\title{
Jet-Cloud Interactions in Compact Steep Spectrum Radio Sources
}

\author{
C. P. O’Dea ${ }^{1}$, W. H. de Vries ${ }^{2}$, A. M. Koekemoer ${ }^{1}$, S. A. Baum ${ }^{1}$, \\ D. J. Axon ${ }^{3}$, P. D. Barthel ${ }^{4}$, A. Capetti ${ }^{5}$, R. Fanti ${ }^{6}$, R. Gelderman ${ }^{7}$, \\ R. Morganti ${ }^{8}$ and C. N. Tadhunter ${ }^{9}$ \\ ${ }^{1}$ Space Telescope Science Institute, 3700 San Martin Drive, Baltimore, MD 21218, USA \\ odea@stsci.edu \\ sbaum@stsci.edu \\ koekemoe@ stsci.edu \\ ${ }^{2}$ Lawrence Livermore National Laboratory, 7000 East Avenue, Livermore, CA 94550-9234, USA \\ wdevries@igpp.ucllnl.org \\ ${ }^{3}$ University of Hertfordshire, College Lane, Hatfield Herts, AL10 9AB, UK \\ dja@star.herts.ac.uk \\ ${ }^{4}$ Kapteyn Astronomical Institute, PO Box 800, 9700 AV Groningen, The Netherlands \\ pdb@astro.rug.nl \\ ${ }^{5}$ Osservatorio Astronomico di Torino, Via Osservatorio 20, I-10025, Pino Torinesse (TO), Italy \\ capetti@to.astro.it \\ ${ }^{6}$ Instituto di Radioastronomia del CNR, Via P. Gobetti, 101, I-40129, Bologna, Italy \\ rfanti@ira.bo.cnr.it \\ ${ }^{7}$ Western Kentucky University, 1 Big Red Way, Bowling Green, KY 42101-3576, USA \\ gelderman@wku.edu \\ ${ }^{8}$ Netherlands Foundation for Astronomy, PO Box 2, 7990 AA Dwingeloo, The Netherlands \\ morganti@nfra.nl \\ ${ }^{9}$ University of Sheffield, Western Bank, Sheffield, S10 2TN, UK \\ C.Tadhunter@sheffield.ac.uk \\ Received 2002 June 30, accepted 2003 January 15
}

\begin{abstract}
We have obtained HST/STIS long slit spectroscopy of the aligned emission line nebulae in three compact steep spectrum (CSS) radio sources - 3C 67, 3C 277.1, and 3C 303.1. We find systematic offsets $\left(\sim 300-500 \mathrm{~km} \mathrm{~s}^{-1}\right)$ of the emission line velocities on one or both sides of the radio sources. We also see evidence for broad lines (FWHM $\sim 500 \mathrm{~km} \mathrm{~s}^{-1}$ ) and complex emission line profiles. In 3C 303.1 the data are consistent with multiple components and possibly split lines. The amplitude of the velocity variations is not so large as to exclude gravitationally-induced motions. However, the complex kinematics, the lack of a signature of Keplerian rotation, and the association of the velocity variations with the radio lobes are consistent with the observed $\sim 300-500 \mathrm{~km} \mathrm{~s}^{-1}$ velocities being driven by the expansion of the radio source. Acceleration of the clouds by the bow shock is plausible given the estimated densities in the clouds and the velocities observed in the much smaller compact symmetric objects and with expansion velocities estimated from spectral ageing. This conclusion is unchanged if we consider the scenario in which the cloud acceleration is dominated by the post bow shock flow.
\end{abstract}

Keywords: galaxies: active — galaxies: jets — galaxies: individual (3C 67, 3C 277.1, 3C 303.1)

\section{Introduction}

The aligned emission line gas in compact steep spectrum (CSS) sources (de Vries et al. 1997, 1999; Axon et al. 2000) provides a diagnostic probe of both the environment of the radio source as well as its propagation. We present results obtained from long slit HST/STIS spectra of three CSS sources - 3C $67(\mathrm{G}, z=0.310), 3 \mathrm{C} 277.1(\mathrm{Q}, z=0.321)$, 3C 303.1 ( $\mathrm{G}, z=0.267)$. We emphasise that these HST observations provide the high spatial resolution necessary to allow us for the first time to examine the detailed kinematics of the nebulae in CSS sources and address the issue of how these sources propagate through their host galaxy. We note that in powerful FR II sources, the ambient clouds will interact with both the bow shock as well as the post bow shock gas. At the present time it is arguable which has the dominant effect on the cloud kinematics. In Section 3, we summarise work from O'Dea et al. (2002) on constraints on the interaction of the clouds with the radio source bow shock. In Section 4, we present a new analysis of the interaction of clouds with the expanding post bow shock gas. We derive similar constraints on the radio source propagation in both cases.

\section{Results}

We have obtained medium dispersion spectroscopy of the [OIII] $\lambda 5007$ line along the radio source axis. The details of the observations are given by O'Dea et al. (2002). The properties of the [OIII] $\lambda 5007$ emission line as a function of distance from the nucleus (along a slit approximately 


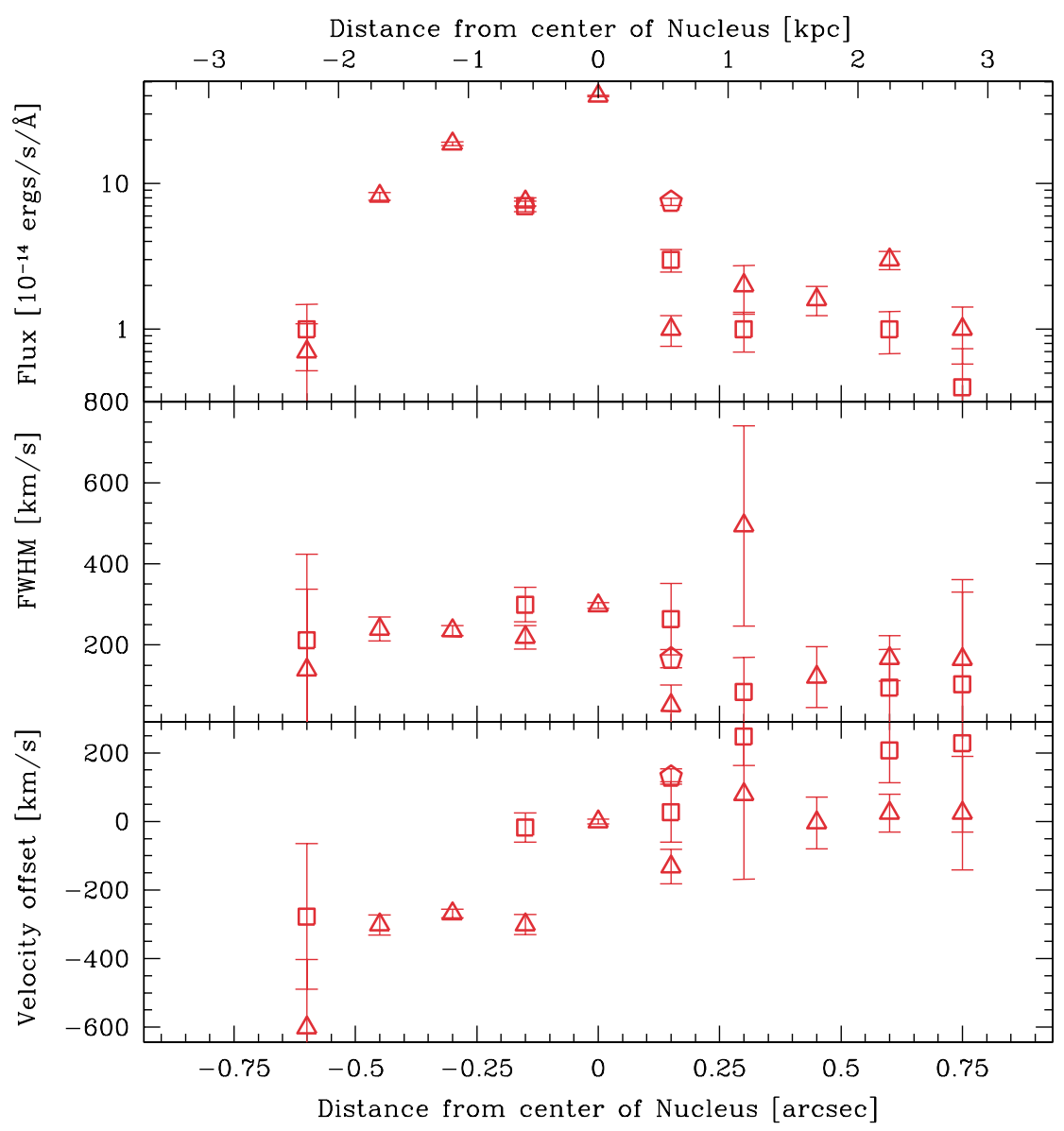

Figure 13 C 67. Plot of [OIII] $\lambda 5007$ emission line properties as a function of distance from the nucleus along a slit approximately parallel to the radio source axis. Top: peak line flux. Middle: FWHM. Bottom: velocity offset from the nucleus. At some locations, multiple Gaussians are necessary to fit the lines. The different components are denoted using different symbols. Positive offset corresponds to north.

along the radio source axis) are shown in Figures 1, 2, and 3. The slit positions are shown in Figure 1 of O'Dea et al. (2002). Our main results are the following.

(1) We find no strong evidence of broad, non-Gaussian components substantially above $\sim 1500 \mathrm{~km} \mathrm{~s}^{-1}$. (2) There is evidence for multiple velocity components in the lines (especially 3C 303.1). (3) There are systematic offsets $\left(\sim 300-500 \mathrm{~km} \mathrm{~s}^{-1}\right)$ of the gas velocities from the systemic (nuclear) velocity. (4) The velocity offsets in the lobes are asymmetric with respect to systemic (nuclear) velocity. (5) The velocity offsets tend to be abrupt, occurring near the nucleus. (6) The FWHM of the lines is $\sim 200-300 \mathrm{~km} \mathrm{~s}^{-1}$ and is not a strong function of position along the source. (7) A comparison with the Baum, Heckman, \& van Breugel (1992) sample reveals that the three CSS sources exhibit kinematics which distinguish them from typical, large, low redshift radio galaxies in the sense that the CSS sources tend to have larger values of the side-to-side velocity difference.

Thus, there is evidence to support the hypothesis that the radio source is interacting with the emission line clouds. Next we discuss the implications of the observations in the context of a scenario in which the bow shock of the radio source runs over ambient clouds, shocking them and accelerating them to velocities of a few hundred $\mathrm{km} \mathrm{s}^{-1}$.

\section{Constraints on the Bow Shock}

A scenario for cloud acceleration by a strong shock is discussed by McKee and collaborators (Klein, McKee, \& Colella 1994). In this picture, the dense clouds co-exist with a hot $T \sim 10^{7} \mathrm{~K}$ intercloud medium. We will apply this model to ambient clouds which are overrun by the radio source bow shock.

The pressure difference across the cloud produces a velocity of

$$
v_{\mathrm{cl}} \simeq 470\left(\frac{v_{\mathrm{b}}}{0.05 c}\right)\left(\frac{\chi}{10^{3}}\right)^{-1 / 2} \mathrm{~km} \mathrm{~s}^{-1},
$$

where $v_{\mathrm{b}}$ is the bow shock expansion velocity and $\chi=n_{\mathrm{cl}} / n_{\mathrm{ISM}}$ is the pre-shock density ratio of the cloud and the ambient medium.

Since we see the line emission covering most of the radio extent of these CSS sources (de Vries et al. 1999), the clouds must cool in a timescale which is less than some fraction $f$ of the age of the radio source. O'Dea et al. (2002) obtain an upper limit to the velocity a cloud could have (for a given cloud density) and still have had 


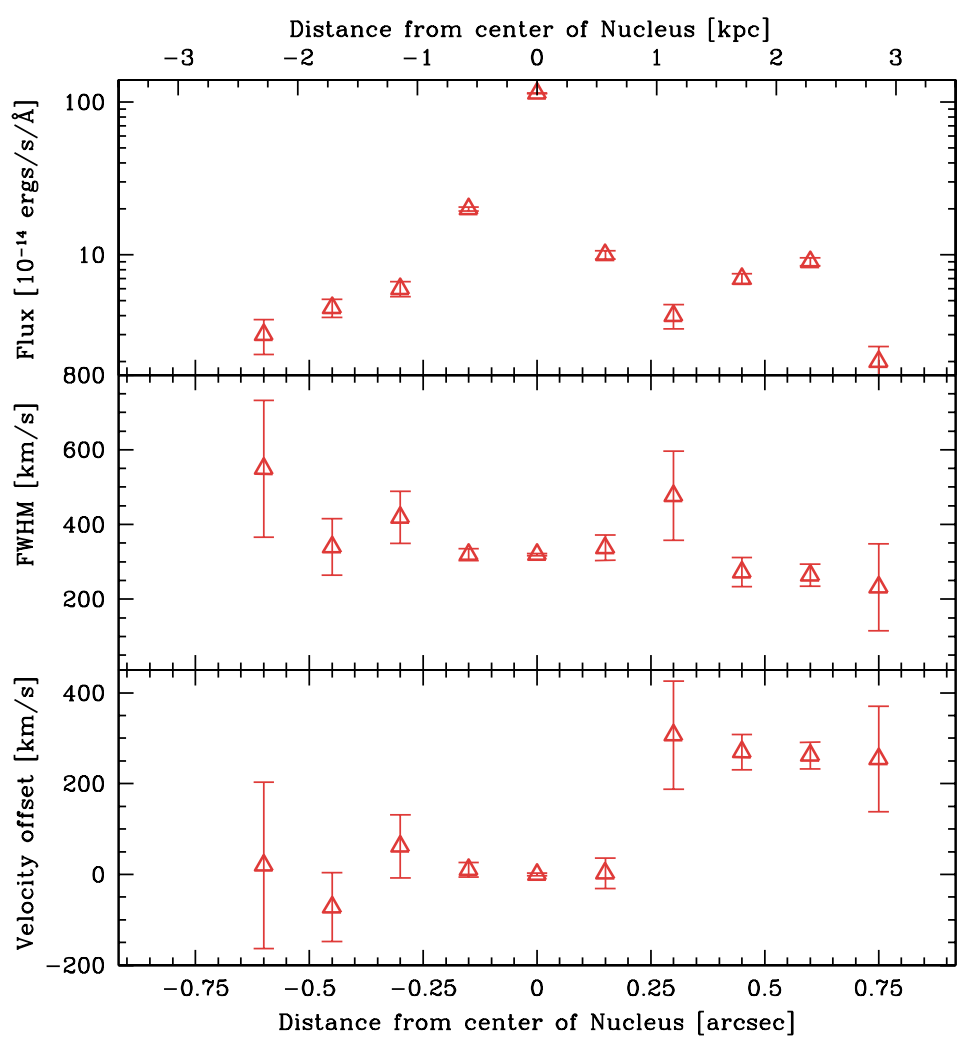

Figure 2 3C 277.1. Plot of [OIII] $\lambda$ 5007 emission line properties as a function of distance from the nucleus along a slit approximately parallel to the radio source axis. Top: peak line flux. Middle: FWHM. Bottom: velocity offset from the nucleus. Positive offset corresponds to northwest.

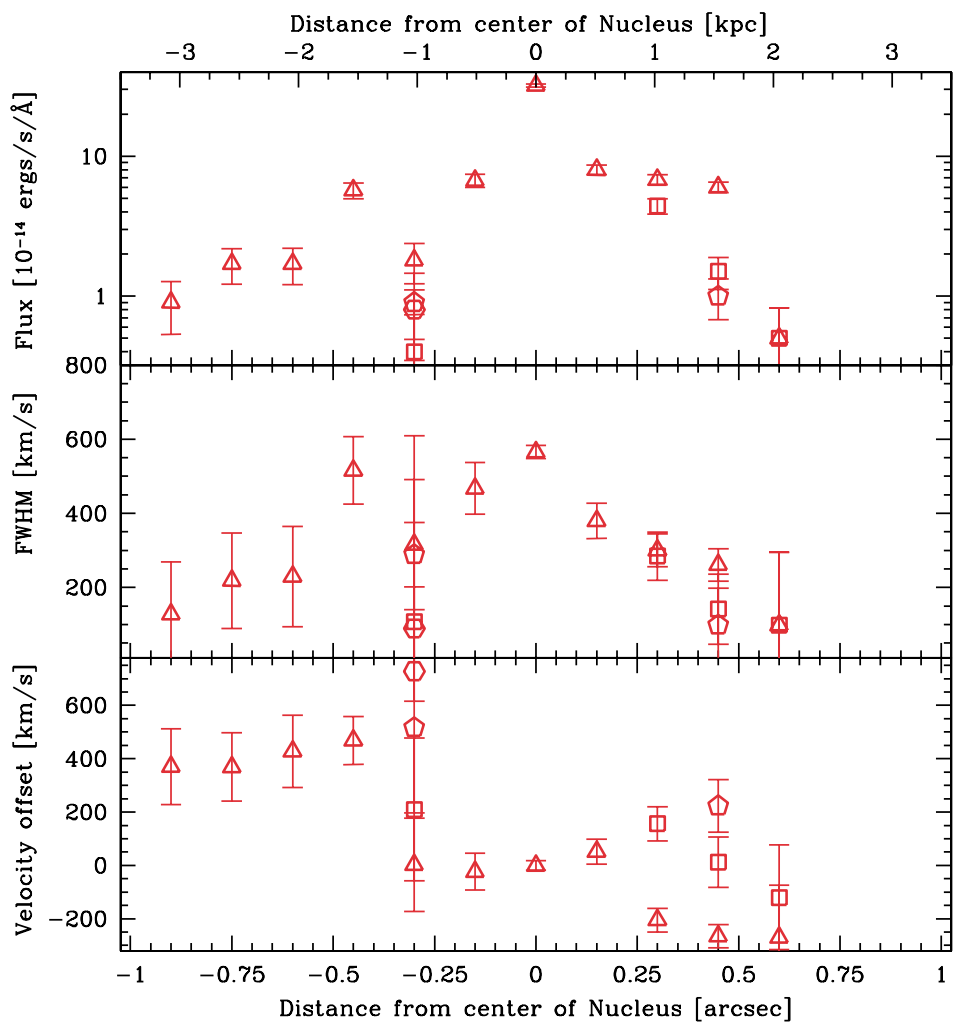

Figure 3 3C 303.1. Plot of [OIII] $\lambda$ 5007 emission line properties as a function of distance from the nucleus along a slit approximately parallel to the radio source axis. Top: peak line flux. Middle: FWHM. Bottom: velocity offset from the nucleus. At some locations, multiple Gaussians are necessary to fit the lines. The different components are denoted using different symbols. Positive offset corresponds to north. 


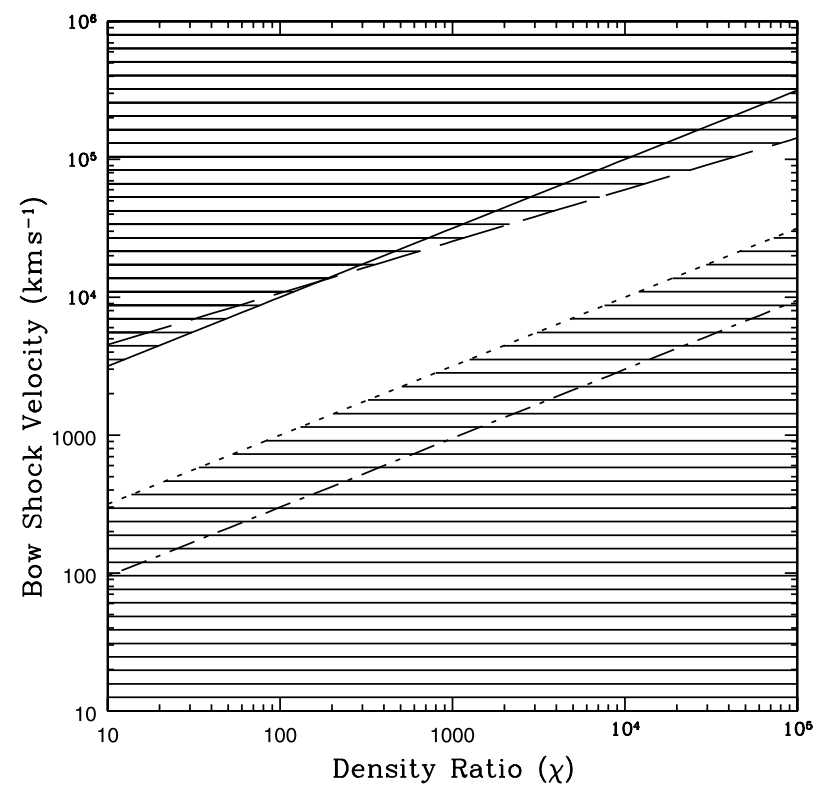

Figure 4 Constraints on bow shock velocity as a function of preshock ratio between cloud and intercloud density $\chi$ assuming that cloud acceleration is dominated by the bow shock. The solid line is the upper limit to $v_{\mathrm{b}}$ from equation (1) assuming that the cloud is accelerated to no more than $1000 \mathrm{~km} \mathrm{~s}^{-1}$, the dotted line is the lower limit to $v_{\mathrm{b}}$ from equation (1) assuming that the cloud is accelerated to at least $100 \mathrm{~km} \mathrm{~s}^{-1}$, the dot-dash line is the lower limit to $v_{\mathrm{b}}$ from equation (1) assuming that the cloud is accelerated to at least $30 \mathrm{~km} \mathrm{~s}^{-1}$ so that the cloud is shock heated to at least $10^{4} \mathrm{~K}$, and the long dashed line is the upper limit to $v_{\mathrm{b}}$ from equation (3) based on the requirement that the cloud's cooling time be less than some fraction of the radio source lifetime. The shaded areas are ruled out by the constraints.

time to cool so as to become detectable, where $n_{\mathrm{cl}}^{\prime}$ is the density in the post-shock cloud and $R$ is the distance from the nucleus to the end of the radio source:

$$
\begin{aligned}
v_{\mathrm{cl}} \lesssim & 760\left(\frac{f}{0.1}\right)^{1 / 3}\left(\frac{R}{5 \mathrm{kpc}}\right)^{1 / 3}\left(\frac{n_{\mathrm{cl}}^{\prime}}{100 \mathrm{~cm}^{-3}}\right)^{1 / 3} \\
& \times\left(\frac{v_{\mathrm{b}}}{0.1 c}\right)^{-1 / 3} \mathrm{~km} \mathrm{~s}^{-1} .
\end{aligned}
$$

O'Dea et al. (2002) also obtain a corresponding upper limit on the bow shock advance velocity which permits the cloud to cool in less than some fraction $f$ of the age of the radio source:

$$
\begin{aligned}
v_{\mathrm{b}} \lesssim & 2.5 \times 10^{4}\left(\frac{f}{0.1}\right)^{1 / 4}\left(\frac{R}{5 \mathrm{kpc}}\right)^{1 / 4}\left(\frac{n_{\mathrm{cl}}^{\prime}}{100 \mathrm{~cm}^{-3}}\right)^{1 / 4} \\
& \times\left(\frac{\chi}{10^{3}}\right)^{3 / 8} \mathrm{~km} \mathrm{~s}^{-1}
\end{aligned}
$$

We see that (1) we do not need to know the age of the radio source, and (2) the unknown quantities are taken to small powers. In Figure 4 we plot equation (3) along with upper and lower limits on $v_{\mathrm{b}}$ from equation (1) assuming upper and lower limits to cloud velocity of 1000 and $100 \mathrm{~km} \mathrm{~s}^{-1}$, respectively. The upper limits on bow shock velocity from the kinematics and the cooling time are in good agreement. The upper and lower limits are consistent with the predicted range of bow shock velocities (from compact symmetric object proper motions and CSS spectral ageing) and with the estimated range of cloud densities from the $[\mathrm{SII}]$ line ratios. Thus, the bow shock acceleration model seems consistent with the existing constraints. The fact that the velocity offsets are relatively constant with distance from the nucleus suggests that the clouds are accelerated to their observed velocities relatively quickly which is also consistent with the bow shock acceleration scenario. Thus, the emission line kinematics are consistent with the view that these CSS sources are young, expanding sources.

\section{Constraints on the Interaction of the Clouds with the Post Bow Shock Gas}

As we have shown above, it is plausible that acceleration by the bow shock is sufficient to fully account for the cloud velocities in sources with high bow shock velocities. However, Villar-Martín et al. (1999) and Best, Longair, \& Röttgering (2000) have suggested that in some radio sources, the acceleration of the emission line clouds is dominated by the hot shocked gas which expands out behind the bow shock.

Here we consider acceleration of the cloud by the outflowing post bow shock gas. Once the bow shock passes over the cloud, the cloud is entrained in the wind-like expanding post-shock medium. The momentum gained by the cloud due to sweeping up the wind is

$$
\Delta m \simeq \pi a^{2} v_{\mathrm{b}}^{2} n_{\mathrm{ISM}}^{\prime} m_{\mathrm{p}} t_{\mathrm{accel}},
$$

where $a$ is the cloud radius, $m_{\mathrm{p}}$ is the proton mass, and $t_{\text {accel }}$ is the timescale over which the cloud is accelerated (e.g., Klein et al. 1994; Best et al. 2000). This gives a cloud velocity

$$
\begin{aligned}
v_{\mathrm{cl}} \simeq & 220\left(\frac{v_{\mathrm{b}}}{0.05 c}\right)^{2}\left(\frac{t_{\mathrm{accel}}}{10^{4} \mathrm{yr}}\right)\left(\frac{a}{1 \mathrm{pc}}\right)^{-1}\left(\frac{\chi}{10^{3}}\right)^{-1} \\
& \times\left(\frac{\delta}{10}\right)^{-1} \mathrm{~km} \mathrm{~s}^{-1}
\end{aligned}
$$

where the post-shock cloud density contrast $\chi^{\prime}$ is larger by the factor $\delta$, i.e., $\chi^{\prime}=\delta \chi$. Here we assume for simplicity that the compression factor $\delta$ is constant, though it is really a function of time in the post-shock cloud due to radiative cooling (e.g., Koekemoer 1996; Dopita \& Sutherland 1996).

An upper limit to $v_{\mathrm{cl}}$ is obtained by requiring that the acceleration timescale $t_{\text {accel }}$ is less than some fraction of the radio source lifetime $f t_{\mathrm{rs}}$. This gives

$$
\begin{aligned}
v_{\mathrm{cl}} \leq & 750\left(\frac{v_{\mathrm{b}}}{0.05 c}\right)\left(\frac{f}{0.1}\right)\left(\frac{R}{5 \mathrm{kpc}}\right)\left(\frac{a}{1 \mathrm{pc}}\right)^{-1}\left(\frac{\chi}{10^{3}}\right)^{-1} \\
& \times\left(\frac{\delta}{10}\right)^{-1} \mathrm{~km} \mathrm{~s}^{-1},
\end{aligned}
$$

where for our illustrative calculations we mainly assume a small value for the radio source size $(5 \mathrm{kpc})$ since these 
sources will mainly interact with dense clouds on the scale of their host galaxy ISM. Thus, if the clouds are accelerated primarily by the post bow shock flow, there is a natural upper limit to the maximum observable cloud velocity which is due to the finite age of the relatively young radio source. However, both $f$ and $v_{\mathrm{b}}$ could easily be larger than we have assumed, raising the upper limit by a factor of at least a few. Note that unlike in equation (2) where the dependence on the parameters is weak, in equation (6) the dependence on the parameters is linear, allowing a stronger test of the scenario.

Klein et al. (1994) estimate that such a cloud is accelerated to a large fraction of the wind velocity in a timescale which is a few times the shock crossing time of the cloud (also called the cloud crushing time)

$$
t_{\mathrm{cc}} \simeq 2.1 \times 10^{3}\left(\frac{a}{1 \mathrm{pc}}\right)\left(\frac{\chi}{10^{3}}\right)^{1 / 2}\left(\frac{v_{\mathrm{b}}}{0.05 c}\right)^{-1} \mathrm{yr} .
$$

The cloud radius is poorly determined, but constraints from $21 \mathrm{~cm} \mathrm{HI}$ measurements in PKS 2322-123 suggest a range $\sim 0.01-10 \mathrm{pc}$ (O’Dea, Baum, \& Gallimore 1994). Taking this range for $a$ and the ranges for $\chi^{\prime}$ and $v_{\mathrm{b}}$ estimated above gives the range for the cloud crushing time $t_{\mathrm{cc}} \sim 10-10^{6} \mathrm{yr}$, where the time is longer for the larger, denser clouds and slower bow shocks. Thus, for most sources, the acceleration time is short compared to the lifetime of the radio source.

If we require that the post bow shock flow accelerate the clouds to a velocity $v_{\mathrm{cl}}=1000 \mathrm{~km} \mathrm{~s}^{-1}$ within a time which is less than some fraction of the radio source lifetime $\left(t_{\text {accel }}<f t_{\mathrm{rs}}\right)$, we obtain a lower limit to the bow shock velocity

$$
\begin{aligned}
v_{b} \gtrsim & 2 \times 10^{4}\left(\frac{a}{1 \mathrm{pc}}\right)\left(\frac{v_{\mathrm{cl}}}{1000 \mathrm{~km} \mathrm{~s}^{-1}}\right)\left(\frac{R}{5 \mathrm{kpc}}\right)^{-1} \\
& \times\left(\frac{f}{0.1}\right)^{-1}\left(\frac{\chi}{10^{3}}\right)\left(\frac{\delta}{10}\right) \mathrm{km} \mathrm{s}^{-1} .
\end{aligned}
$$

We assume that the clouds are heated and ionised by the bow shock. The shocks heat the clouds to the temperature

$$
T_{\mathrm{cl}} \simeq 1.1 \times 10^{5}\left(\frac{v_{\mathrm{cl}}}{100 \mathrm{~km} \mathrm{~s}^{-1}}\right)^{2} \mathrm{~K},
$$

(e.g., Taylor, Dyson, \& Axon 1992; Sutherland, Bicknell, $\&$ Dopita 1993). An approximation to the cooling time of the shocked gas is

$$
t_{\mathrm{cool}} \sim 1 \times 10^{3}\left(\frac{n_{\mathrm{cl}}^{\prime}}{100 \mathrm{~cm}^{-3}}\right)^{-1}\left(\frac{T_{\mathrm{cl}}}{10^{6}}\right)^{3 / 2} \mathrm{yr},
$$

(e.g., Taylor et al. 1992), and we have used $n_{\mathrm{cl}}^{\prime}$ to denote densities in the post-shock cloud.

Klein et al. (1994) suggest that the clouds will be subsequently destroyed by instabilities generated in the post bow shock wind on a timescale similar to the cloud crushing time. Note that Klein et al. (1994) consider non-radiative clouds. However, the clouds in these radio

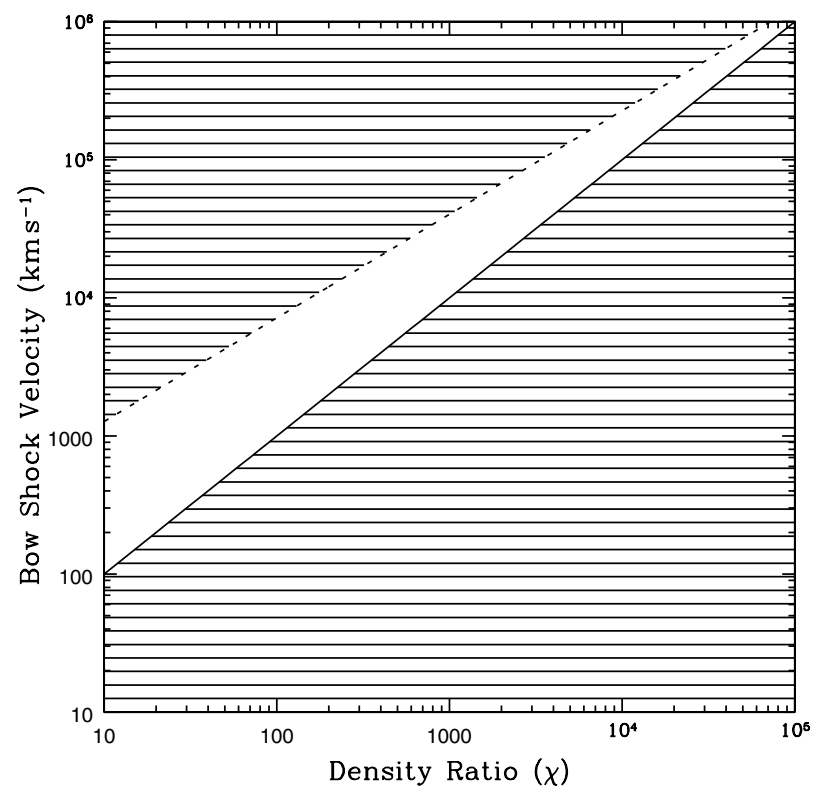

Figure 5 Constraints on bow shock velocity $v_{\mathrm{b}}$ as a function of density contrast $\chi$ assuming that the clouds are accelerated by the post bow shock flow. The solid line is the lower limit on $v_{\mathrm{b}}$ from equation (8), the dotted line is the upper limit on $v_{\mathrm{b}}$ from equation (11). These constraints assume $v_{\mathrm{cl}}=500 \mathrm{~km} \mathrm{~s}^{-1}, f=0.1, \zeta=10$, $a=1 \mathrm{pc}, \delta=10$, and $n_{I S M}=0.1 \mathrm{~cm}^{-3}$. The shaded area is ruled out by the constraints.

galaxies are clearly cooling and radiating. The increase in density due to the cooling will tend to increase the cloud lifetime (equation (7)). Thus, the cloud lifetime against shredding will be larger than $t_{\mathrm{cc}}$ by some factor $\zeta$.

We require that the cloud cooling time is less than the cloud destruction time (i.e., $t_{\text {cool }}<\zeta t_{\mathrm{cc}}$ ), so that the clouds are visible in emission lines before they are destroyed. Then, from equations (10) and (7) we obtain the following upper limit to the bow shock velocity:

$$
\begin{aligned}
v_{b} \lesssim & 4.1 \times 10^{4}\left(\frac{a}{1 \mathrm{pc}}\right)^{1 / 4}\left(\frac{\zeta}{10}\right)^{1 / 4}\left(\frac{\chi}{10^{3}}\right)^{1 / 2} \\
& \times\left(\frac{n_{\mathrm{cl}}^{\prime}}{10^{3} \mathrm{~cm}^{-3}}\right)^{1 / 4} \mathrm{~km} \mathrm{~s}^{-1} .
\end{aligned}
$$

Note that there are several additional parameters in the post bow shock flow scenario compared with the bow shock scenario so it should be easier to find a suitable region of parameter space. In Figure 5 we show the constraints on bow shock velocity from equations (8) and (11) for a potentially plausible choice of parameters. We see that for this choice of parameters the post bow shock flow is able to produce the observed cloud velocities for plausible values of the bow shock velocity. We note that there is considerable overlap in the range of required bow shock velocity in the bow shock only (Fig. 4) and post bow shock flow (Fig. 5) scenarios. Thus, adopting the post bow shock wind scenario does not necessarily alter the conclusion that these CSS radio sources are expanding rapidly and are likely to be young sources. 


\section{Acknowledgments}

Support for this work was provided by NASA through grant number GO-08104.01-97A from the Space Telescope Science Institute, which is operated by the Association of Universities for Research in Astronomy, Inc., under NASA contract NAS5-26555. WDV's work was performed under the auspices of the US Department of Energy, National Nuclear Security Administration by the University of California, Lawrence Livermore National Laboratory under contract no. W-7405-Eng-48. We are grateful to the referees for helpful comments.

\section{References}

Axon, D. J., Capetti, A., Fanti, R., Morganti, R., Robinson, A., \& Spencer, R. 2000, AJ, 120, 2284
Baum, S. A., Heckman, T. M., \& van Breugel, W. 1992, ApJ, 389, 208

Best, P. N., Longair, M. S., \& Röttgering, H. J. A. 2000, MNRAS, 311,23

de Vries, W. H., et al. 1997, ApJS, 110, 191

de Vries, W. H., O’Dea, C. P., Baum, S. A., \& Barthel, P. D. 1999, ApJ, 526, 27

Dopita, M. A., \& Sutherland, R. S. 1996, ApJS, 102, 161

Klein, R., McKee, C., \& Colella, P. 1994, ApJ, 420, 213

Koekemoer, A. M. 1996, PhD thesis, Australian National University O’Dea, C. P., et al. 2002, AJ, 123, 2333

O’Dea, C. P., Baum, S. A., \& Gallimore, J. F. 1994, ApJ, 436, 669

Sutherland, R. S., Bicknell, G. V., \& Dopita, M. A. 1993, ApJ, 414,510

Taylor, D., Dyson, J. E., \& Axon, D. J. 1992, MNRAS, 255, 351

Villar-Martín, M., Tadhunter, C., Morganti, R., Axon, D., \& Koekemoer, A. 1999, MNRAS, 307, 34 